\title{
Research and Development of Testing Device for Evaluating Force Transmission and Grasping Pressure of Laparoscopic Forceps
}

\author{
Rui Zhu, ${ }^{1 *}$ Maxime Maréchal, ${ }^{2}$ Masahiro Suetsugu, ${ }^{3}$ Ikuo Yamamoto, ${ }^{3}$ \\ Murray J. Lawn, ${ }^{1,2}$ Keitaro Matsumoto, ${ }^{4}$ and Takeshi Nagayasu ${ }^{4}$ \\ ${ }^{1}$ Medical-Engineering Hybrid Professional Development Program, Graduate School of Biomedical Sciences, \\ Nagasaki University, 1-12-4 Sakamoto, Nagasaki 852-8523, Japan \\ ${ }^{2}$ Yamamoto Lab., Graduate School of Engineering, Nagasaki University, \\ 1-14 Bunkyo, Nagasaki 852-8521, Japan \\ ${ }^{3}$ Yamamoto Lab., Program of Mechanical Engineering, Engineering Department, Nagasaki University, \\ 1-14 Bunkyo, Nagasaki 852-8521, Japan \\ ${ }^{4}$ Graduate School of Biomedical Sciences, Medical and Dental Sciences, Nagasaki University, \\ 1-12-4 Sakamoto, Nagasaki 852-8523, Japan
}

(Received May 1, 2019; accepted October 21, 2019)

Keywords: laparoscopic forceps, grip force, grasping force, contact pressure distribution, force transmission

In this study, we have developed a device with the aim of understanding the relationship between the force exerted by a surgeon on the handles/grips of laparoscopic forceps and the force transmitted to the forceps jaw side. To measure this relationship, force is applied to the handles/grips of a set of laparoscopic forceps using an electrically powered actuator and a pressure sensor load cell is used to measure the force applied. On the jaw side, a forcesensitive resistor (FSR) generates pressure information. A microprocessor uses the load cell data, and a proportional-integral-derivative (PID) controller stably maintains the actuation pressure to match that requested by the user's settings. A user interface has been developed for this device to allow both engineers and surgeons to use it. To date, two types of forceps have been analyzed, both Olympus $\mathrm{HiQ}+$ series graspers, and the mechanical force transmission mechanisms involved have been described. The pressure distribution exerted on the jaw side has also been obtained using a Fujifilm Prescale ${ }^{\circledR}$ system (FPS).

\section{Introduction}

Minimally invasive surgery (MIS) has become widely accepted worldwide. Since 1987, when the first modern laparoscopic surgery was successfully performed in France, it has been recognized as an approach with major benefits to patients by the international community. MIS has continued to see rapid development, involving large numbers of surgeons and engineers who have joined together to research and develop this new approach to surgery. This cycle

*Corresponding author: e-mail: zhur1008@gmail.com

https://doi.org/10.18494/SAM.2019.2419 
of research and development has continued to accelerate the acceptance and standardization of MIS to date. The typically quoted advantages of laparoscopic surgery include reduced post-operation trauma, reduced blood loss during operations, reduced hospital stays, and the facilitation of remote surgery. From the beginning of this century, specific types of MIS have included natural-orifice transluminal endoscopic surgery (NOTES) using a natural aperture of the human body rather than creating a surgical wound, ${ }^{(1)}$ and single-port laparoscopic or single-incision surgery (SPL/SIS), focusing on centralizing all of the instruments into one port/incision-typically the umbilicus. ${ }^{(2,3)}$ In addition, surgical robots that can help surgeons perform operations remotely such as da Vinci ${ }^{\circledR}$ and robot-assisted laparoscopic surgery (RALS) have received extensive attention in the second decade of the 21st century, and the number of associated applications is increasing year by year. ${ }^{(4,5)}$

Although these approaches to laparoscopic surgery are significant, providing increased efficiency to meet increasing demand and positive feedback for the global research community, they require specialized surgical training over and above that required for open surgery. There are many aspects to be considered when training for laparoscopic surgery. Firstly, the operation is conducted while viewing a video monitor of the surgical site in real time and, depending on the specific surgery, the monitor will often provide a reverse image of the operating area, requiring the surgeon to control the instruments in a mirror-inverted manner. ${ }^{(6,7)}$ Secondly, haptic feedback is degraded when using laparoscopic instruments, especially graspers, when compared with open surgery. ${ }^{(8-11)}$ On the basis of current clinical data and research on graspers, ${ }^{(12-15)}$ the authors have observed that different companies produce graspers with different types of handles and jaws, and that some surgical experts prefer to use new product series or switch to products manufactured by different companies, despite the absence of information on the products such as diagrams showing force transmission between the handle side and the jaw side, and the lack of jaw pressure distribution data.

The goal of this study is to develop a device that can evaluate graspers with regard to the relationship of the force between the handles and the jaw and the pressure distribution data at the jaw. This could provide surgeons with a reference guide for the use of specific graspers for training as well as in preparation for operations.

\section{Materials and Methods}

The newly developed device consists of three main parts, namely, mechanical parts, hardware, and software; the device is mounted with an Olympus $\mathrm{HiQ}+{ }^{\circledR}$ series grasper, as shown in Fig. 1. In this study, an Olympus A60201A handle "Ergo S" was used. Note that Olympus offers several types of jaws, which are interchangeable; local surgeons have indicated a preference for a Johann forceps jaw (Olympus A64120A), owing to its good working visibility, and this jaw was therefore used in this study. The shaft length from the handle to the jaw is 330 $\mathrm{mm}$ for a regular model of these forceps. A thin short universal type of grasping forceps jaw (Olympus WS1744/1) was also used to provide a comparison for differentiation and therefore optimize the selection of forceps in relation to the target task with respect to force transmission. A schematic diagram of the grasper is shown in Fig. 2. Currently produced forceps tend to 


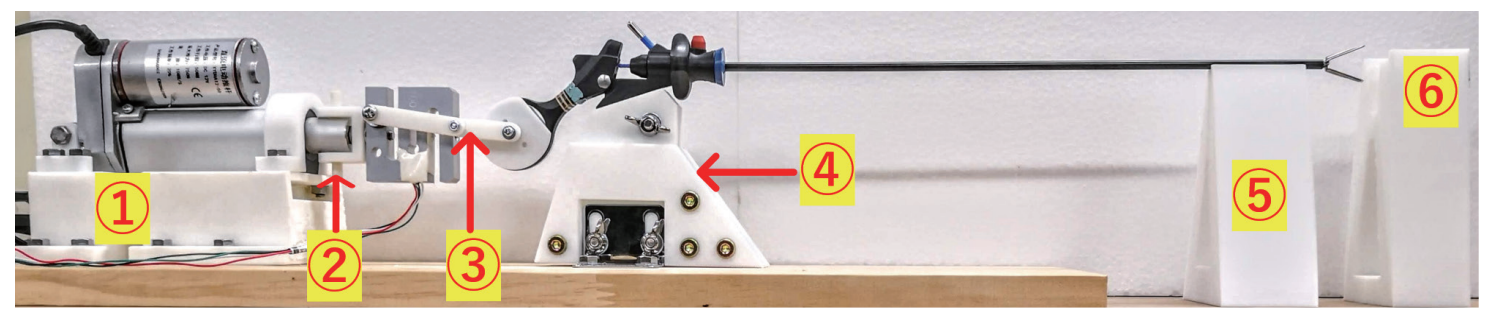

(a)

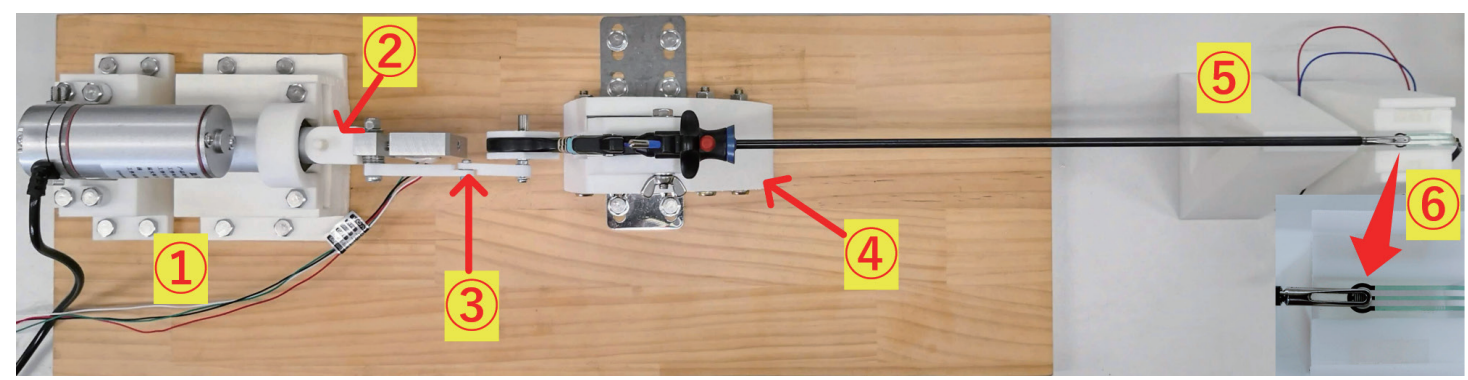

(b)

Fig. 1. (Color online) Overall view of test device mounted with an Olympus HiQ $+{ }^{\circledR}$ series grasper: (a) front and (b) top sides. The main driving actuator is mounted on two blocks (1), the actuator is linked to the load cell sensor (2), a return only articulated link is connected between the load cell sensor and the movable side of the forceps handle (3), the fixed part of the forceps handle is clamped by a receptor tailored to provide the maximum stability of the forceps, which is also mounted on the base (4), and a triangular stander behind the jaw of forceps is supported by the shaft against flexion by gravity (5). The forceps tip/jaw is also mounted to prevent slight oscillation and, finally, the FSR is also independently mounted (6)

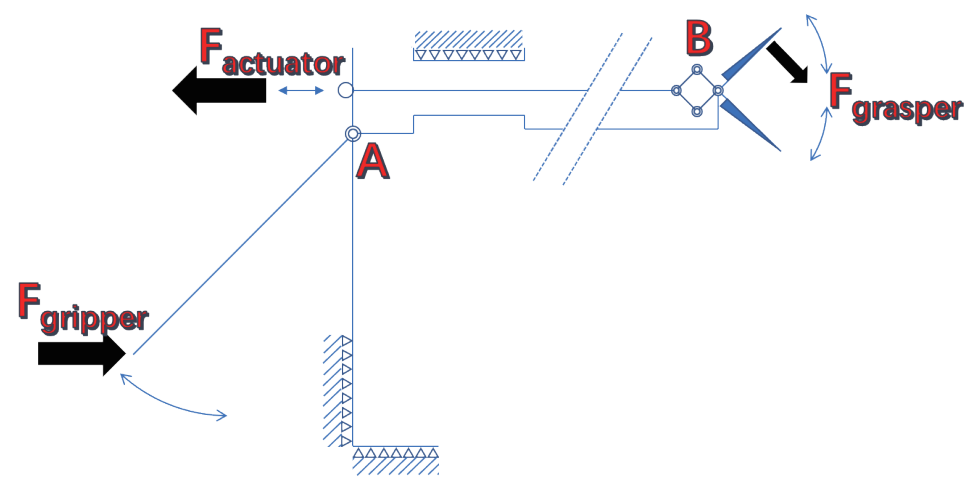

Fig. 2. (Color online) Mechanical schematic diagram of the forceps.

change the type of motion in two ways. Firstly, it can be seen from Fig. 2 that the handle and shaft convert the force from a circular motion (hand grip force) to a reciprocating motion at point A. Then, the force converts the reciprocating motion (linear actuation) of the shaft back to a circular motion at the connection of the shaft and jaw (point B, jaw grip force).

\subsection{Mechanical description}

To simulate a surgeon's grip on the forceps, we designed a base mount to hold the forceps as well as provide an actuator, a force feedback sensor, and an associated control system. Most 
of the parts - in white - were designed by CAD and 3D-printed. A mechanical schematic diagram of the forceps is shown in Fig. 2. An overall view of the mounted test device is shown in Fig. 1; the main driving actuator is mounted on two blocks (1), the actuator is linked to the load cell sensor (2), a return only articulated link is connected between the load cell sensor and the movable side of the forceps handle (3), the fixed part of the forceps handle is clamped by a receptor tailored to provide the maximum stability of the forceps, which is also mounted on the base (4), and a triangular stander behind the jaw of forceps is supported by the shaft against flexion by gravity (5). The forceps tip/jaw is also mounted to prevent slight oscillation and, finally, the FSR is also independently mounted (6).

\subsection{Hardware}

The control system circuit schematic is shown in Fig. 3. The control system is built around a Raspberry $\mathrm{Pi}^{\circledR} 3$ Model $\mathrm{B}+$, which was chosen for its high processing speed [Cortex-A53 (ARMv8) 64-bit SoC@1.4 GHz]. The actuator provides a maximum force of $750 \mathrm{~N}$ over a $50 \mathrm{~mm}$ stroke $(10 \mathrm{~mm} / \mathrm{s} \max , 20 \%$ duty cycle) with a $12 \mathrm{~V} \mathrm{DC}$ power source. The motor is pulse width modulation (PWM)-controlled to maintain the required force. The force exerted by the actuator is measured using an S-type load cell (100 kg, 0.03\% accuracy) connected to an HX711 analog-to-digital converter (ADC) (four wire types). The pressure information is transmitted on a 1-WIRE bus with a sample rate of $10-80 \mathrm{~Hz}$. A force-sensitive resistor (FSR) is used to measure the force exerted at the forceps jaw end. An Arduino ${ }^{\circledR}$ Due slave was used to provide high-resolution ADC functionality to digitize the FSR data after amplification using an op-amp $(0-5 \mathrm{~V})$. The FSR pressure data is then interpreted using the FSR datasheet. The equation for the output voltage from the circuit can be expressed as

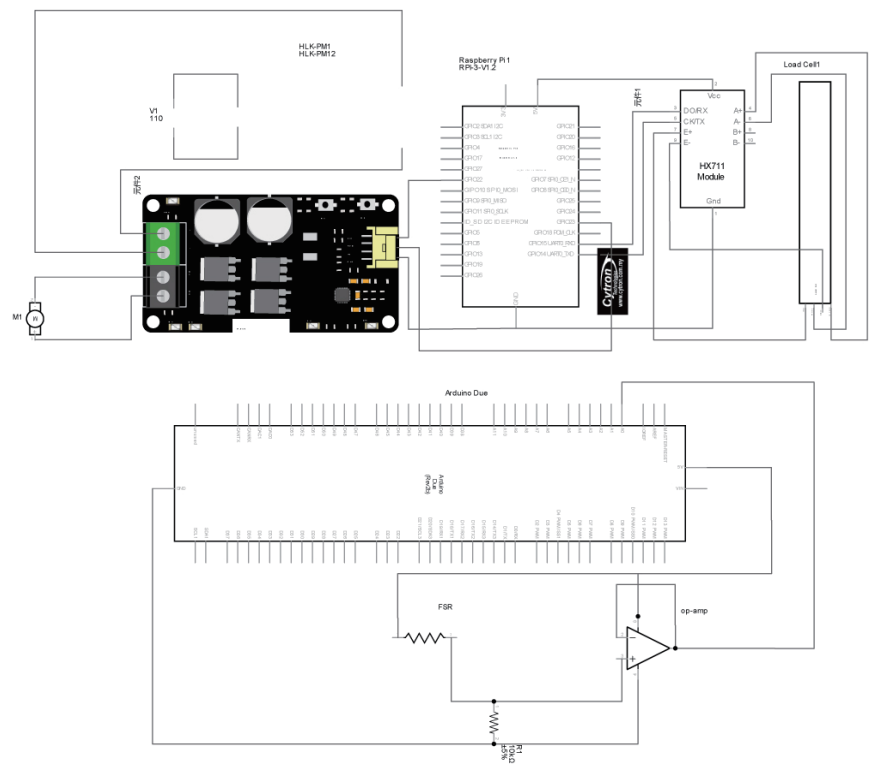

Fig. 3. (Color online) Circuit diagram of the device. 


$$
V_{\text {out }}=\frac{V_{+}}{1+\frac{R_{F S R}}{R_{M}}} .
$$

Here, $V_{+}$is the input voltage and $R_{M}$ is the resistance of the resistor connected between the output side of the FSR and the ground.

\subsection{Software}

This device can be used by both engineers and surgeons. A user-friendly touchscreen-based interface was developed to use the GUI interface intuitively. A flowchart of the software is shown in Fig. 4.

Following the main thread built with Python version 3.5.2 using a PyTK library, users can set up a target force in gram-force (gf) and start a proportional-integral-derivative (PID) controller thread to automatically drive the movable handle to the desired location of the target force (gf). Also, to generate pressure distribution information at the jaw with the target force (gf), a timer option provides a countdown of 5 or $120 \mathrm{~s}$ test for a Fujifilm Prescale ${ }^{\circledR}$ system (FPS). This option sets a timer, then closes the PID autocontrol thread. After the PID controller thread has been closed completely, the timer option thread sends a signal to the actuator to move to the initial position to release the jaw. Another two threads that are started within the program initiation are a load cell data process thread and an FSR data process thread to allow the continued reading and logging of data.

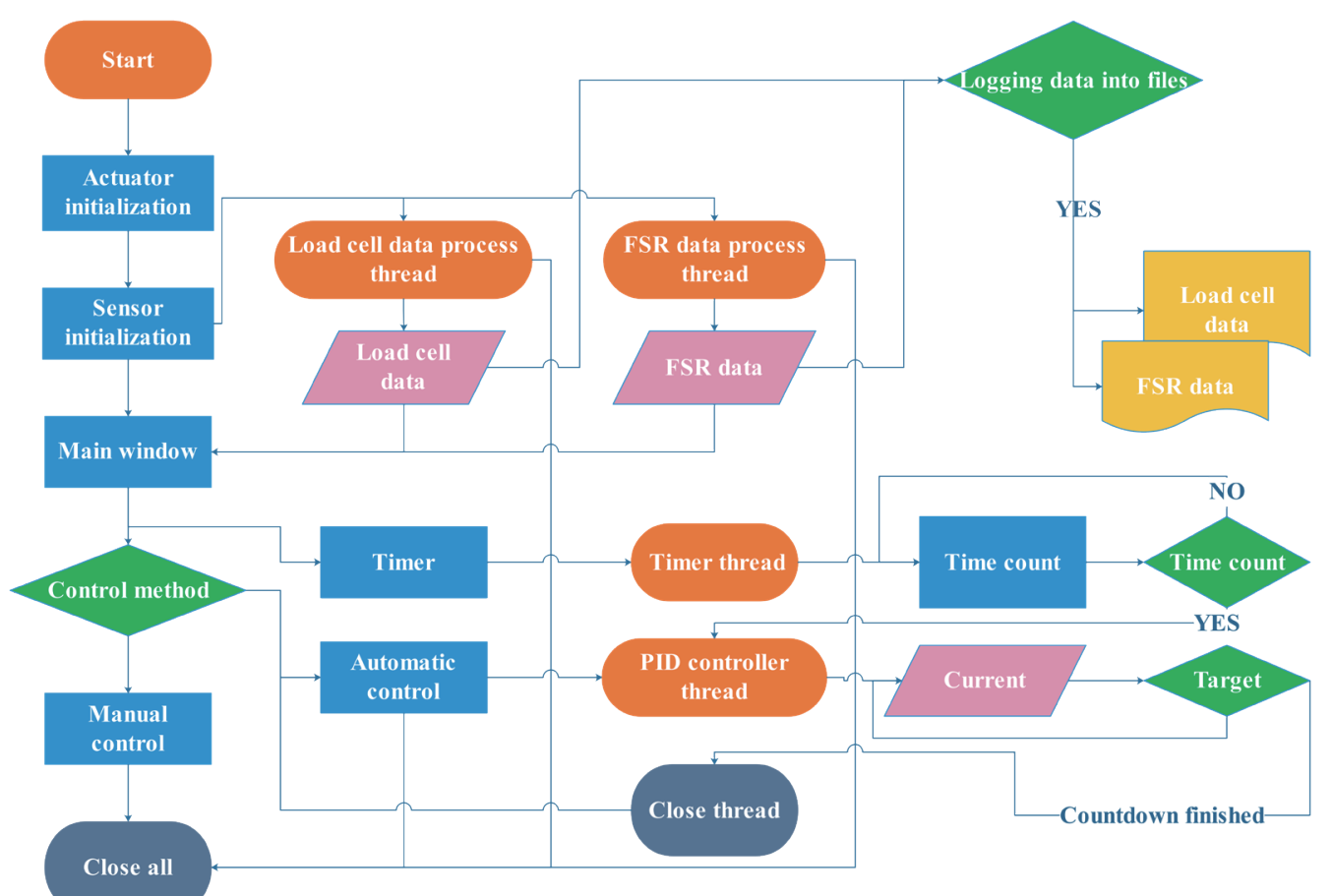

Fig. 4. (Color online) Control program flowchart. 


\subsection{Tests}

In this study, we used two methods for testing graspers using this device. One of the methods used the sensors that were mounted on the device to obtain pressure forces from the jaw side in the range of 100 to $1200 \mathrm{gf}$ at $100 \mathrm{gf}$ intervals; this process was fully automated.

The other method was based on FPS constant-pressure testing by using LLLW (ultrasuper low pressure) and 4LW (extremely low pressure) sensitive films; this method focused on the pressure distribution of the jaw with the same force (gf) ranges to show the characteristics unique to each type of laparoscopic forceps, particularly high-pressure hotspots. The two types of forceps tested are shown in Fig. 5.

\section{Results}

The pressure distribution data was scanned and processed in the FPS software. We built a Python 3 library to read and process the exported data and calculated the pressure distribution per pixel, which was then displayed using a colormap jet.

\subsection{Force transmission}

The results of the pressure data for the Johann-type forceps are shown in Fig. 6, where the $x$-axis is time and the $y$-axis is the pressure force shown in gf.

The input force on the hand grip side is shown in Fig. 7, and the relationship between the output force and the input force can be approximated as

$$
F_{\text {output }}=F_{\text {input }}+G
$$

Here, $G$ is an almost constant parameter providing a stable gain in the case of the Johann-type forceps.

In the case of the thin short universal type forceps, the results of pressure data for the thin short universal forceps are shown in Fig. 8 and the input force on the hand grip side is shown in Fig. 9. Also, the force transmission is the same as that for the Johann-type forceps and can also

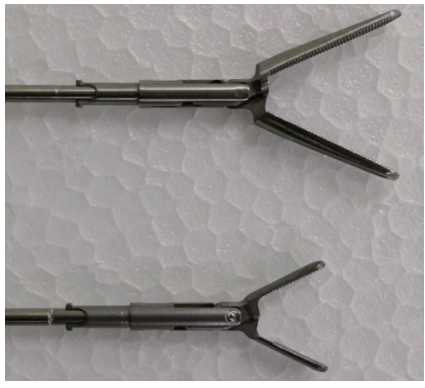

(a)

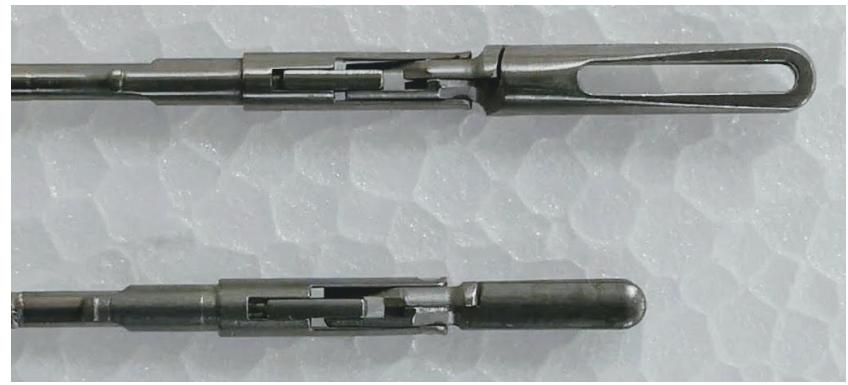

(b)

Fig. 5. (Color online) Forceps: (a) side and (b) plan views (Johann-type forceps above and thin short universal forceps below). 
Pressure force [gf]

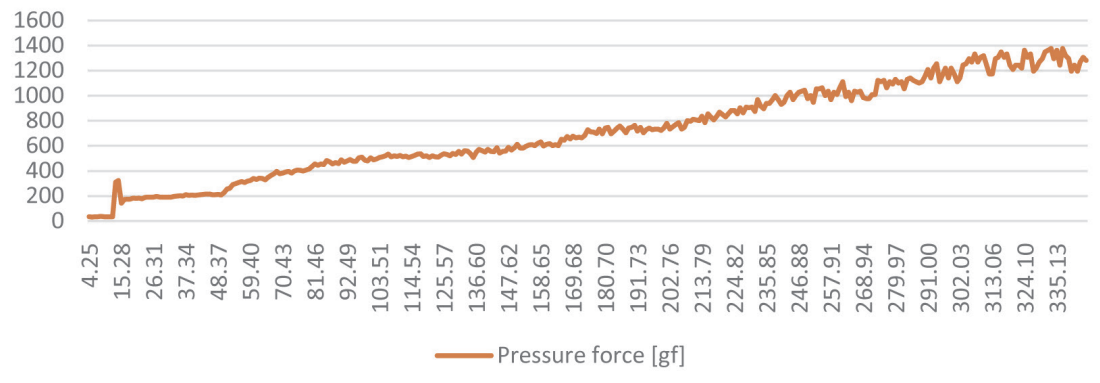

Fig. 6. (Color online) Johann-type forceps pressure data.

Grip force [gf]

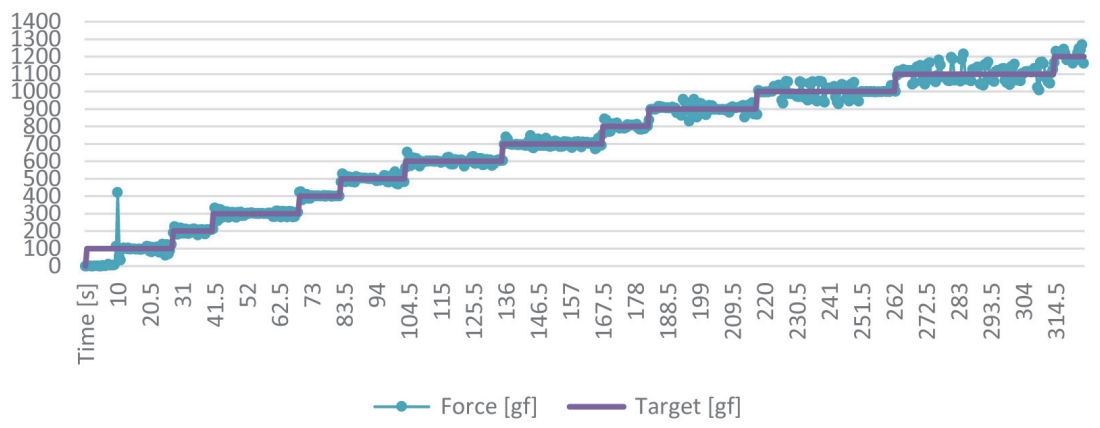

Fig. 7. (Color online) Input force data obtained on the hand grip side of Johann-type forceps.

Pressure force [gf]

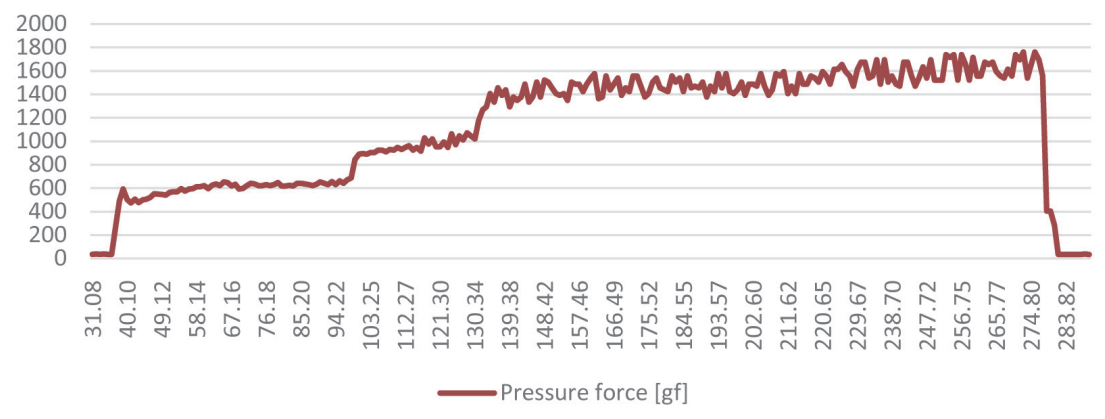

Fig. 8. (Color online) Thin short universal forceps pressure data.

Grip force [gf]

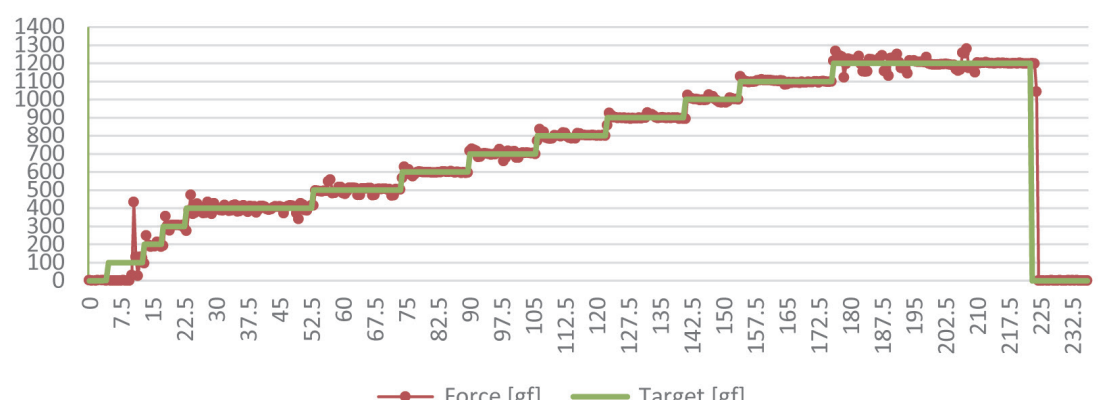

Fig. 9. (Color online) Input force data obtained on the hand grip side of thin short universal forceps. 
be calculated using Eq. (2), but $\mathrm{G}$ is greater than that for the Johann-type forceps. Because of its smaller and thinner jaw structure, the pressure at the jaw with a low gripping force under $500 \mathrm{gf}$ is twice that of the Johann-type forceps.

\subsection{Pressure distributions}

The resulting FPS-based pressure distributions for the Johann-type and thin short universal forceps are respectively shown in Figs. 10 and 11. The two figures clearly show the characteristics of both forceps including high-pressure hotspots. This type of visual pressure information will be useful for medical staff, particularly surgeons.
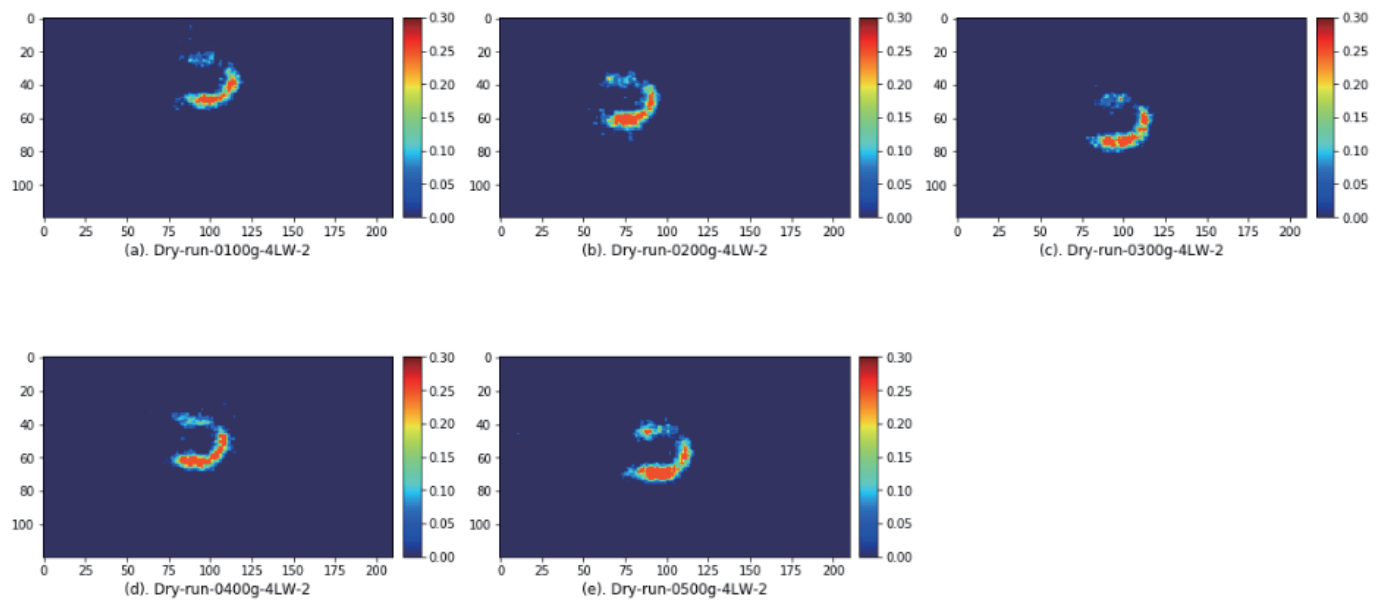

(a)
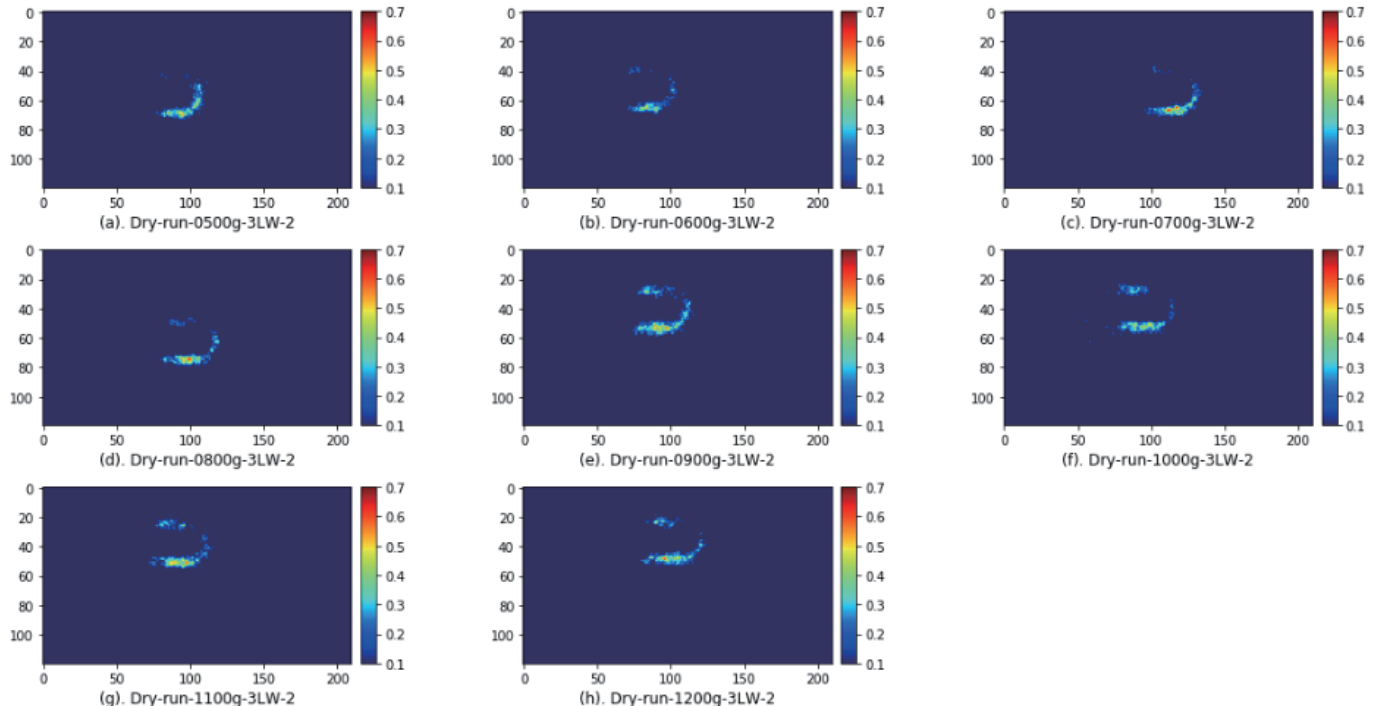

(b)

Fig. 10. (Color) Pressure distributions of Johann-type forceps jaw: (a) data obtained using 4LW film and (b) results of tests using LLLW film. 

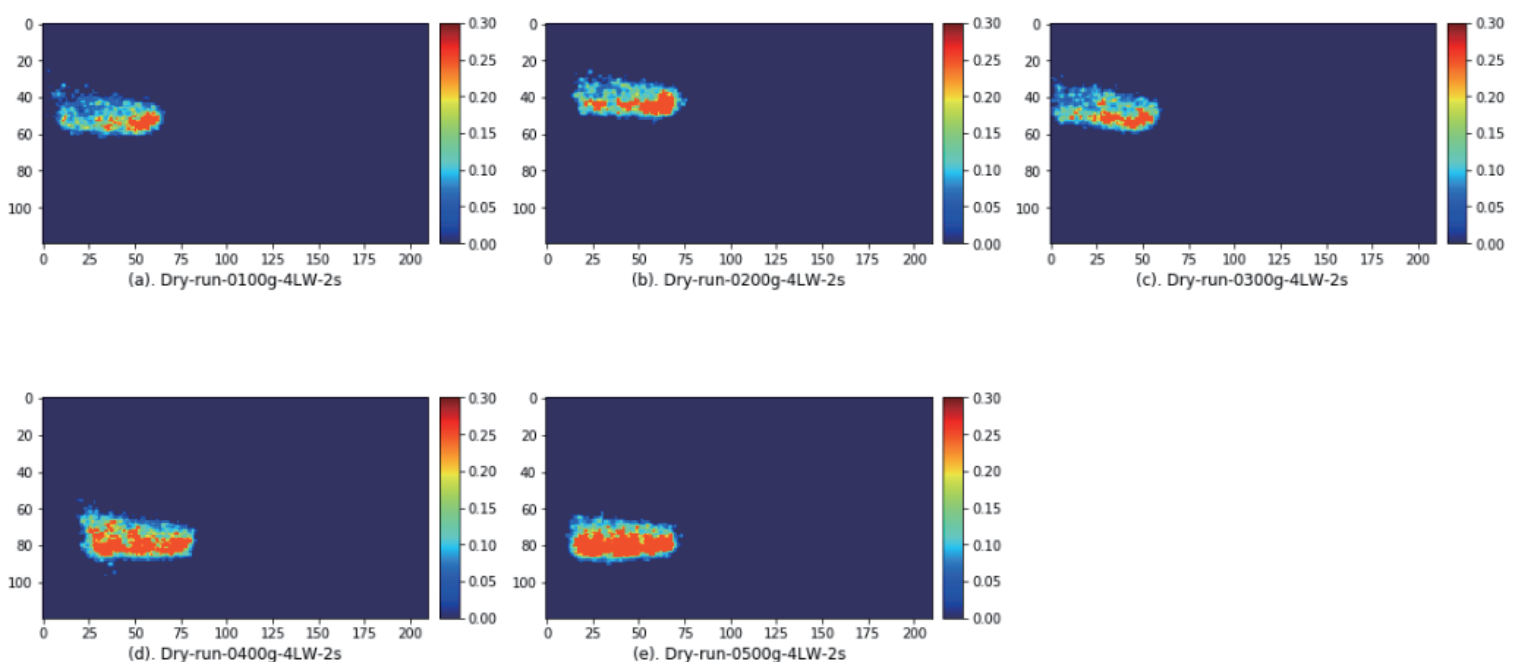

(a)
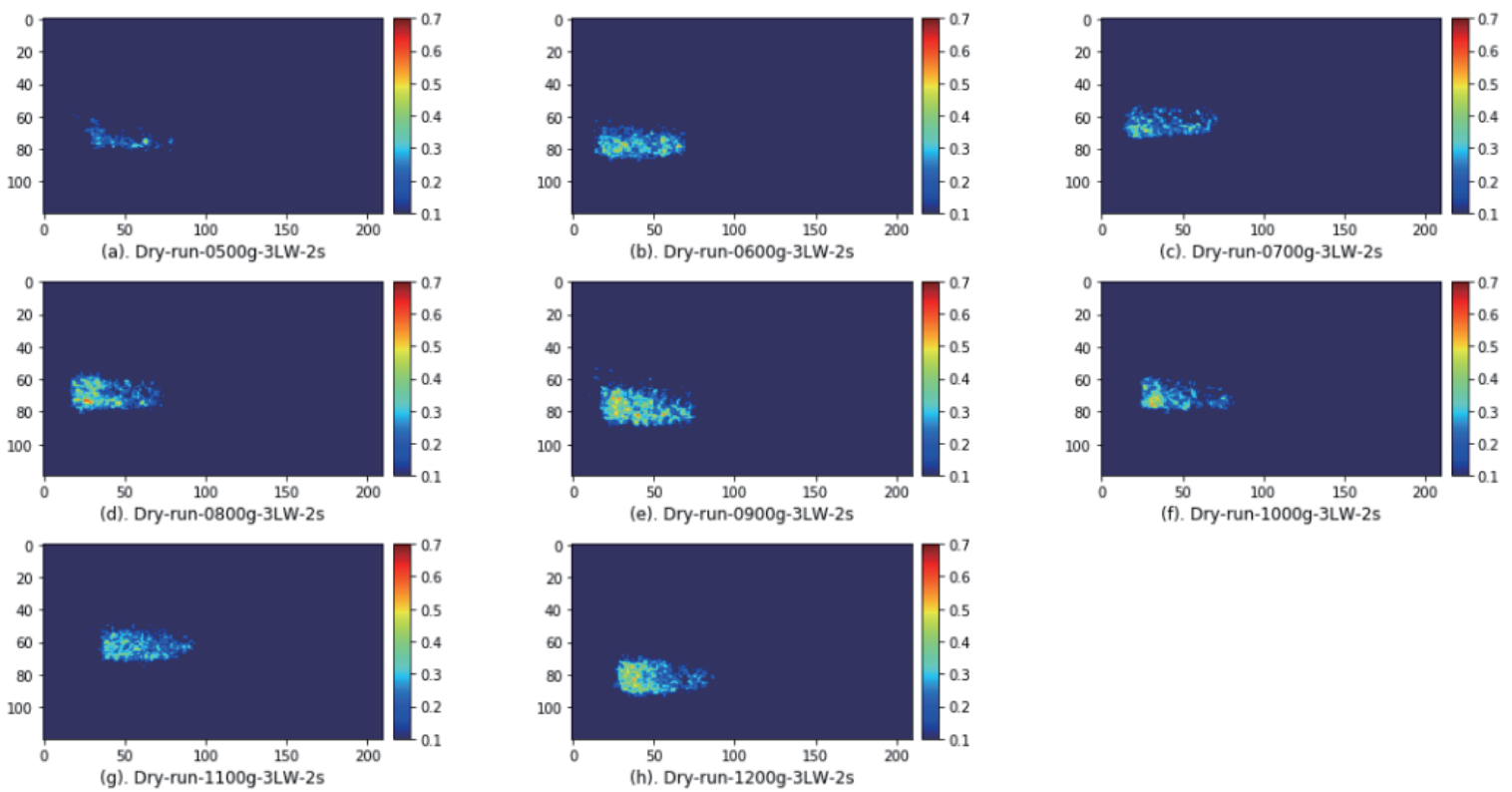

(b)

Fig. 11. (Color) Pressure distributions of thin short universal forceps jaw: (a) data obtained using 4LW film and (b) results of tests using LLLW film.

\section{Discussion}

We have presented a device for evaluating the relationship between the hand grip force applied to the handle and the resulting grasping force and contact pressure distribution at the jaw of conventional laparoscopic surgery forceps during operation by using an FSR and a force-sensitive film. According to Figs. 7 and 9, the grip force acts dynamically on the handle side. The target input force (hand grip force) is detected by the load cell spikes at the instant the forceps jaws make contact with the FSR (the state change from no load to a load). This signal occurs because of the feedback PID controller, which is used in this study as a linear 
controller, and a status change is caused by a nonlinear signal. This evaluation was corrected by using both the FSR sensor and load cell data; in this study, a 120 s FPS procedure was used to evaluate the contact pressure distribution. The test data from the $5 \mathrm{~s}$ FPS procedure was unstable during the transition from no load to a load.

As the target force increases, current/force oscillations occur between the load cell and the FSR sensor in the range of 900 to $1200 \mathrm{gf}$ for the Johann-type forceps and in the range of 1000 to $1200 \mathrm{gf}$ for the thin short universal type forceps. This phenomenon is most likely caused by the actuator mechanism due to the $\mathrm{CW}$ and $\mathrm{CCW}$ actuation switching hysteresis combined with the limitations of actuator control. Specifically, the motor is PWM-controlled, and the minimum functional actuation is $20 \%$ (PWM duty cycle). This clearly makes fine control very difficult, resulting in oscillation that becomes more notable as the force increases. Despite the initial spike and the mechanical vibration occurring during high output levels, a relatively stable data is obtained in this analysis, which can be used as a basis for future work.

\section{Conclusions}

We have developed a device for testing laparoscopic forces to better understand the grasping pressure applied to the forceps jaw. By using a load cell, FSR, and FPS, we obtained the forces transmitted and pressure distribution data of two types of forceps. Using this data, surgeons can make better-informed choices regarding their choice of forceps for any given task and be better prepared for any given surgery. Furthermore, the use of the data may increase training efficiency.

\section{References}

1 T. H. Baron: Br. J. Surg. 94 (2007) 1. https://doi.org/10.1002/bjs.5681

2 R. Tacchino, F. Greco, and D. Matera: Surg. Endosc. 23 (2009) 896. https://doi.org/10.1007/s00464-008-0147-y

3 J. H. Kaouk, G. P. Haber, R. K. Goel, M. M. Desai, M. Aron, R. R. Rackley, C. Moore, and I. S. Gill: Urology 71 (2008) 3. https://doi.org/10.1016/j.urology.2007.11.034

4 M. J. Mark: JAMA 285 (2001) 568. https://doi.org/10.1001/jama.285.5.568

5 S. T. Chen, M. C. Wu, T. C. Hsu, D. W. Yen, C. N Chang, W. T. Hsu. C. C. Wang, M. Lee, S. H. Liu and C. C. Lee: J. Surg. Oncol. 117 (2018) 497. https://doi.org/10.1002/jso.24867

6 L. P. Aitchison, C. K. Cui, A. Arnold, E. Nesbitt-Hawes, and J. Abbott: Surg. Endosc. 30 (2016) 5068. https:// doi.org/10.1007/s00464-016-4855-4

7 S. Bernhardt, S. A. Nicolau, L. Soler, and C. Doignon: Med. Image Anal. 37 (2017) 66. https://doi.org/10.1016/ j.media.2017.01.007

8 T. Horeman, F. Delft, M. D. Blikkendaal, J. Dankelman, J. J. Dobbelsteen, and F. W. Jansen: Surg. Endosc. 28 (2014) 1961. https://doi.org/10.1007/s00464-014-3425-x

9 D. Smit, E. Spruit, J. Dankelman, G. Tuijthof, J. Hamming, and T. Horeman: Surg. Endosc. 31 (2017) 299. https://doi.org/10.1007/s00464-016-4972-0

10 A. Araki, K. Makiyama, H. Yamanaka, D. Ueno, K. Osaka, M. Nagasaka, T. Yamada, and M. Yao: Surg. Endosc. 31 (2017) 1999. https://doi.org/10.1007/s00464-016-5153-x

11 İ. M. Koç, T. Eray, B. Sümer, and N. Çerçi: Tribol. Int. 100 (2016) 317. https://doi.org/10.1016/j.triboint.2016.03.001

12 D. Jones, A. Jaffer, A. A. Nodeh, C. S. Biyani, and P. Culmer: J. Endourol. 32 (2018) 529. https://doi. org/10.1089/end.2017.0894

13 R. Hernandez, A. Onar-Thomas, F. Travascio, and S. Asfour: Surg. Endosc. 31 (2017) 4805. https://doi. org/10.1007/s00464-017-5558-1

14 K. Miyashita, T. O. Vrielink, and G. Mylonas: Int. J. Comput. Assist. Radiol. Surg. 13 (2018) 659. https://doi. org/10.1007/s11548-018-1717-7

15 M. Valdis, M. W. A.Chu, C. Schlachta, and B. Kiaii: J. Thorac. Cardiovasc. Surg. 151 (2016) 1498. https://doi. org/10.1016/j.jtcvs.2016.02.016 